\title{
Reply to the Comment by Silvano Romano [1] on the Paper [2] ${ }^{1}$
}

\author{
I. M. Stanković, V. M. Marković, and Lj. Z. Kolar-Anić \\ Faculty of Physical Chemistry, University of Belgrade, Studentski trg 12-16, 11158 Belgrade, P.O. Box 47, Serbia \\ e-mail:stankoviciv@gmail.com,wladimirmarkowic@gmail.com, ljiljana.kolar.anic@gmail.com
} Received April 22, 2013

DOI: $10.1134 / \mathrm{S} 0036024413120339$

It is true that this totally open ensemble (which is not-generalized ensemble) had already been discussed in the literature, but never with this specific derivation. This derivation is direct, it starts from the discussion of $N, V, E$ reservoirs, thus being especially suitable for physicochemists. Our paper also contains a suitable table of partition sums and thermodynamic potentials.

Our results coincide with that given in the literature [3]. Starting from the grand canonical ensemble, we can derive the statistical sum of a totally open system using the second postulate of statistical thermodynamics [3]:

$$
\Pi(T, P, \mu)=\sum_{j} \Xi(T, V, \mu) \exp \left(\frac{-P V_{j}}{k_{\mathrm{B}} T}\right),
$$

where $\Pi$ is the totally open partition sum and $\Xi$ the grand canonical partition sum:

$$
\Xi(T, V, \mu)=\sum_{i} \sum_{k} \exp \left(\frac{\mu N_{k}-E_{i}}{k_{\mathrm{B}} T}\right) .
$$

\footnotetext{
${ }^{1}$ The article is published in the original.
}

Thus, the statistical sum of a totally open system is:

$$
\Pi(T, P, \mu)=\sum_{i} \sum_{j} \sum_{k} \exp \left(\frac{\mu N_{k}-E_{i}-P V_{j}}{k_{\mathrm{B}} T}\right),
$$

and if considering the degeneration or statistical weight, the previous term becomes:

$$
\begin{gathered}
\Pi(T, P, \mu)=\sum_{i} \sum_{j} \sum_{k} g\left(E_{i}, V_{j}, N_{k}\right) \\
\quad \times \exp \left(\frac{\mu N_{k}-E_{i}-P V_{j}}{k_{\mathrm{B}} T}\right) .
\end{gathered}
$$

\section{REFERENCES}

1. Silvano Romano Russ. J. Phys. Chem. A. 87 (12), (2013).

2. I. M. Stanković, V. M. Marković, and Lj. Z. KolarAnić Russ. J. Phys. Chem. A. 85 (13), 2257 (2011).

3. A. Münster, Statistical Thermodynamics (Springer, Berlin Heidelberg New York, Academic Press, New York, London, 1969), vol. 1, ch. 3, sects. 3.1 and 3.2. 